\title{
Effect of the Strain Kind on the Texture and Microstructure of Low-Alloyed Steel
}

\author{
N. M. Shkatulyak, V. V. Usov, E. S. Savchuk, E. A. Dragomeretskaya, and D. V. Bargan \\ South Ukrainian National Pedagogical University Named after K.D. Ushinsky, 26 Staroportofrankovskaya Street, \\ Odessa 65020, Ukraine \\ Correspondence should be addressed to N. M. Shkatulyak; shkatulyak@mail.ru
}

Received 11 April 2016; Revised 15 June 2016; Accepted 26 June 2016

Academic Editor: Hyoung Kim

Copyright (C) 2016 N. M. Shkatulyak et al. This is an open access article distributed under the Creative Commons Attribution License, which permits unrestricted use, distribution, and reproduction in any medium, provided the original work is properly cited.

Crystallographic texture and microstructure of low-alloyed steel after twist extrusion (TE) and subsequent cold rolling along and across the TE axis were studied. The double axial cylindrical texture with axes $\langle 110\rangle$ and $\langle 100\rangle$ parallel to the TE axis and the vortexlike microstructure are formed in the steel during the TE. The subsequent rolling of extruded steel along the TE axis promotes the forming of typical steel rolling texture as well as the microstructure with elongated grains in rolling direction. Typical steel rolling texture as well as the equiaxed microstructure is formed in extruded steel after rolling in the direction transverse to the TE axis. The mechanisms of formation of the texture are discussed.

\section{Introduction}

Methods of severe plastic deformation (SPD), such as torsion under high pressure (HPT), 3D forging, equal-channel angular pressing (ECAP), accumulative roll bonding (ARB), cyclic extrusion and compression (CEC), repetitive corrugation and straightening (RCS), and twist extrusion (TE), are increasingly used recently for production of bulk ultrafine grained (UFG) materials [1]. Most popular kinds of metal semifinished in mechanical engineering such as precision long profiles including profiles with a cylindrical axial channel can be prepared only by the method of TE [2]. Certain structural aspects of unique properties of bulk UFG materials and prospects of their applications are presented in [1]. A significant number of works have been devoted to aspects of the texture formed during ESCAP [3-6], ARB [7-9], and HPT [10]. Crystallographic texture of aluminum alloy and bcc steel after TE has been investigated in [11]. Texture formation during the TE occurs through activation of slip systems as well as by means of vortex motion of refinement grains, which is similar to the turbulent liquid flow.

Control of the texture and anisotropy caused by it of physical and mechanical properties of structural materials is one of main tasks of modern metallurgy. Developing technologies to create an optimal texture in sheets can serve as an important reserve for improving the properties of products in many ways. Such technology processes typically include a combination of different kinds of plastic deformation and heat treatment. For example, the bar or rod with a circular or square cross section is the final product obtained using ECAP. This form is suitable when using the forging operations, but not suitable for obtaining of sheets and plates. In order to overcome this lack of ECAP, in [12] the combination of ECAP with the conventional rolling of the $\mathrm{Fe}-\mathrm{Co}-\mathrm{V}$ alloy is investigated. Similar processing applied to aluminum alloy 8112 [13], as well as for commercial Al-Mg alloy [14]. In the last three above cited papers changes in the structure of alloys and mechanical properties are studied in detail, but the texture is disregarded.

The microstructure and crystallographic texture of alloy Al-2.5 wt.\% Mg processed by combining accumulative roll bonding and conventional rolling have been studied in [15]. Evolution of crystallographic texture during ECAP processing followed by drawing was analyzed in [16]. Evolution of crystallographic texture during ECAP followed by hot rolling was performed in [17].

Texture is the main cause of mechanical properties anisotropy of polycrystalline bodies as was mentioned above [18]. Study of possibility influence on the texture of the workpiece subjected to the TE by the subsequent 


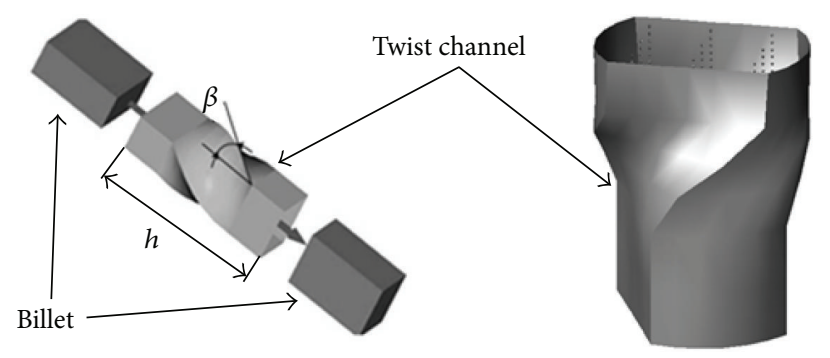

FIGURE 1: Circuit of processing by twist extrusion [20].

deformation that is differed from the TE is important. It can be rolling or hydrostatic extrusion, for example, for lengthy profiles. Elongation of profile along its axis is the main type of deformation in these processes. Simple shear in a plane perpendicular to the extrusion axis is the basic form of the deformation during the TE [2]. Effect of the $\mathrm{TE}$ and consequent rolling in directions along and across the TE axis of aluminum alloy AA6063 was investigated in [19].

The aim of this work is to study the texture of lowalloyed steel at two stages of deformation. Steel samples on a first deformation stage were subjected to the TE. On a second stage of deformation steel samples were subjected to consequent cold rolling both along and across the extrusion axis.

\section{Materials and Methods}

Cast cylindrical billets of low-carbon steel (C, $0.14 \%$; Mn, $1.66 \%$; S, 0.035\%; P, 0.035\%; Ni, 0.30\%; Cr, 0.30\%; Cu, 0.30\%) were as starting material. The blanks were placed in a matrix $[1,2]$ with a helical channel (with parameters $\beta_{\max }=60^{\circ}$ and $h=50 \mathrm{~mm}$ ). The cross section of the workpiece was perpendicular to the pressing axis and constant along the axis. The initial angle $\gamma$ of helix inclination to the compression axis is zero. During the TE process this angle varies along matrix height $h$ (Figure 1) [20].

Detailed description of the equipment is given in [20]. The treatment was performed under the following parameters:

(i) Heating temperature of the container and the matrix for the steel was $673 \mathrm{~K}$.

(ii) Strain rate was $3 \mathrm{~mm} / \mathrm{s}$.

(iii) Strain rate was $0.2 \div 1\left(\mathrm{c}^{-1}\right)$.

(iv) Pressure of the TE was $200 \mathrm{MPa}$.

(v) Workpiece size: cross section was $18 \times 28 \mathrm{~mm}$ and length was $100 \mathrm{~mm}$.

During the TE process two passes of steel workpiece were carried out. After completion of the TE from steel billets $5 \mathrm{~mm}$ thick were cut for the subsequent rolling along the extrusion axis and perpendicular to its axis and appropriate samples for texture studies. Rolling was performed at room temperature on a laboratory rolling mill with diameter of mill rolls $180 \mathrm{~mm}$. The rolling was carried out with small reduction $(\approx 3-5 \%)$ up to 56 and $80 \%$ reduction of thickness, which corresponds to 0.82 and 1.6 of the true logarithmic strain $(\varepsilon)$.

Crystallographic texture of the metal was studied by Xrays method with the construction of inverse pole figures (IPFs) [21]. Texture of extruded samples was examined in the direction perpendicular and parallel to the extrusion axis. Texture of samples after consequent rolling was investigated in the normal direction (ND) to the rolling plane. Before the study of texture, the samples were chemically polished to the depth of $0.1 \mathrm{~mm}$ to remove the distorted surface layer. Profiles of diffraction lines of textured and withouttexture samples were recorded in equal conditions by the $\theta-2 \theta$ scanning on the diffractometer DRON-3M with the focusing by Bragg and Brentano. As pole density ratio of integrated intensities of diffraction lines of textured samples to intensities of corresponding diffraction lines of sample without texture was used. Normalization according to Morris [22] was used when constructing IPFs. Pole density on the IPF is proportional to the ratio of the line (hkl) intensity of the textured sample to the intensity of corresponding line of sample without texture:

$$
P_{\mathrm{hkl}}=\frac{I_{\mathrm{hkl}}^{T}}{I_{\mathrm{hkl}}^{0}} K,
$$

where the normalization factor $K$ is

$$
K=\frac{1}{\sum_{\Delta} A_{\mathrm{hkl}} I_{\mathrm{hkl}}^{T} / I_{\mathrm{hkl}}^{0}},
$$

where $A_{\mathrm{hkl}}$ is the fraction of total area associated with the normal to the plane $(\mathrm{hkl})$ on the plane projection. The individual areas can be determined graphically by means of a polar planimeter. The sum $A_{\mathrm{hkl}}$ must be equal to one (i.e., $\left.\sum_{\Delta} A_{\mathrm{hkl}}=1\right)$. In this way

$$
P_{\mathrm{hkl}}=\frac{I_{\mathrm{hkl}}^{T} / I_{\mathrm{hkl}}^{0}}{\sum_{\Delta} A_{\mathrm{hkl}} I_{\mathrm{hkl}}^{T} / I_{\mathrm{hkl}}^{0}},
$$

where the integrated intensity of line $\{h \mathrm{kl}\}$ in textured samples is $I_{\mathrm{hkl}}^{T} ; I_{\mathrm{hkl}}^{0}$ is the same for the sample without texture.

Metallographic structure was investigated in the rolling plane by means of metallographic microscope MIM-7 using the camera VEB-E-TREK DEM 200 to output the image structure on the computer monitor. 


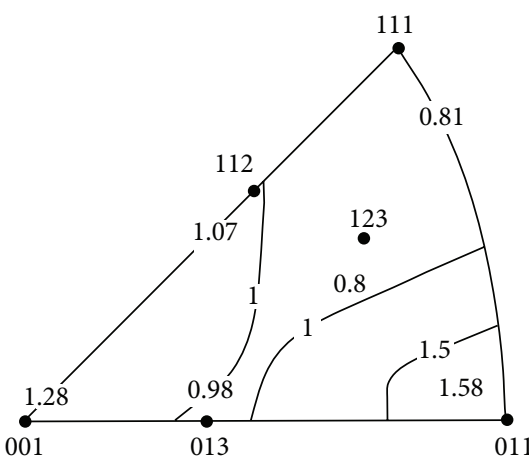

(a)

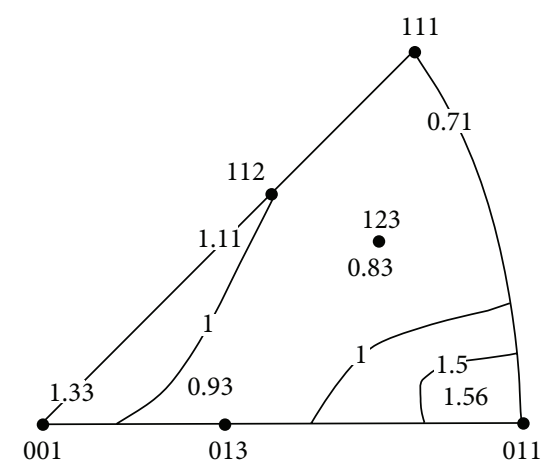

(b)

Figure 2: Experimental IPFs of steel after TE parallel (a) and perpendicular (b) to the axis of TE.

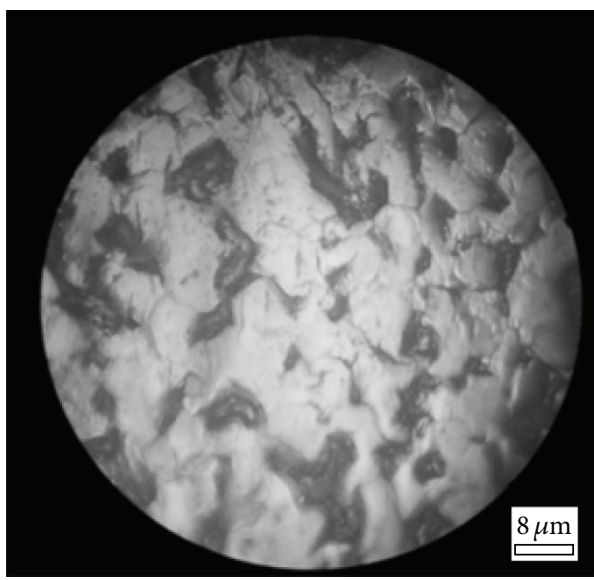

(a)

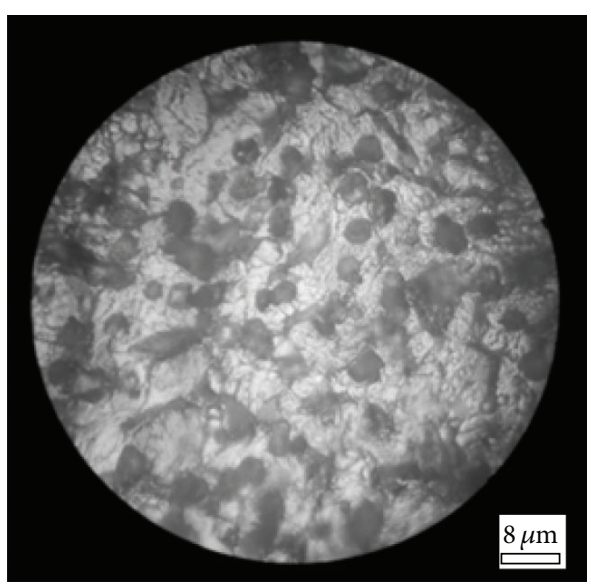

(b)

FIGURE 3: The microstructure of extruded steel in 2 passes: (a) parallel to the extrusion axis; (b) perpendicular to the extrusion axis.

\section{Results and Discussions}

3.1. Twist Extrusion. Experimental IPFs after TE of steel are shown in Figure 2.

The maximal value of pole density 1.58 is observed in the pole $\langle 110\rangle$ on the IPF of direction parallel to the TE axis (Figure 2(a)). Maximal pole density (1.56) corresponds also to the pole $\langle 110\rangle$ on the IPF of direction perpendicular to the TE axis (Figure 2(b)). The second largest pole density corresponds to the $\langle 100\rangle$ pole (resp., 1.28 and 1.33).

Figure 3 shows the microstructure of extruded steel. Traces of vortex-strain are traced. Images of the microstructure show that grain boundaries are tortuous and have tend to curl.

Orientations of crystals relative to the texture axis are completely disordered when typical axial texture is formed, as seen in the example of idealized axial texture $\langle 100\rangle$ (Figure 4).

Radial direction of sample always coincides with one of specific crystallographic directions when the so-called "cylindrical" crystallographic texture is formed as schematically shown in Figure 5.

Comparison of experimental IPFs (Figure 2) with theoretical pole figures (Figures 4 and 5) allows us to conclude that the distribution of crystal orientations in Figure 1 is specific and can be described as a double "cylindrical" texture with axial directions of $\langle 110\rangle$ and $\langle 100\rangle$ parallel to the extrusion axis ( $X$ in Figures 4 and 5 ).

For example, the cylindrical texture was detected in niobium wire produced by hot pressing (swage wire) at true strain of 2.3. Directions $\langle 110\rangle$ were parallel to the wire axis. Planes $\{001\}$ were placed parallel to the wire surface [23]. It was also shown that the cylindrical texture formed usually during the production of wires from the metal with a bodycentered cubic lattice [24].

Forming cylindrical axial texture of the above type was established previously in bcc alloy of Fe-Al system after extrusion through a cylindrical die at sufficiently high temperatures $\left(850^{\circ} \mathrm{C}-1300^{\circ} \mathrm{C}\right)$ [25]. Formation of the aforementioned cylindrical axial texture can be explained, for example, in such a way [25]. In an axially symmetric stretch in all cases $\langle 110\rangle$ fibrous texture develops. In grains with axis of stretching [011] there are directions [100] and [011] that are perpendicular to [011] axis (Figure 5). These orthogonal directions mechanically behave differently. Grains with fiber axis $\langle 110\rangle$ will deform mostly by the pure shear, that is, at least by plane strain. However, since the total deformation of 


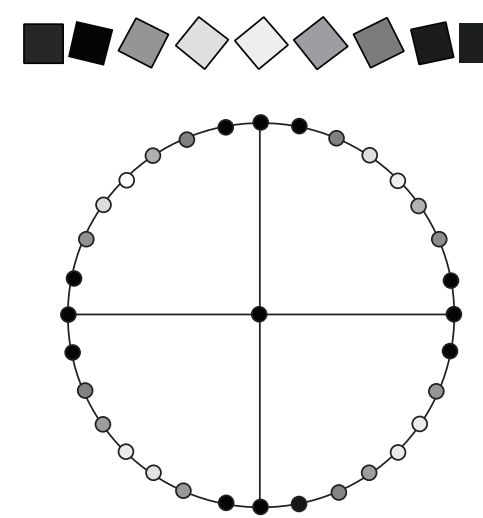

(a)

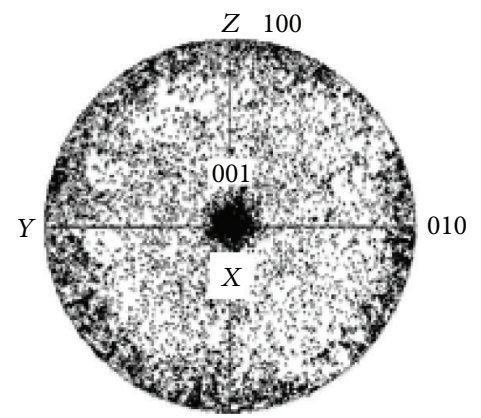

(b)

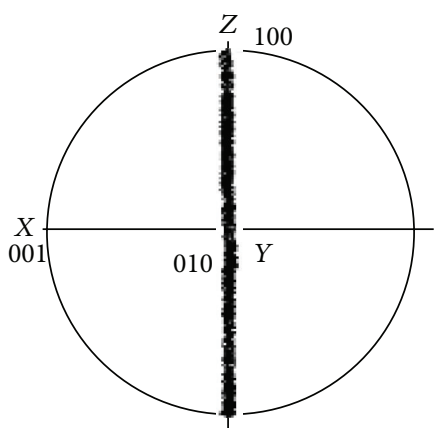

(c)
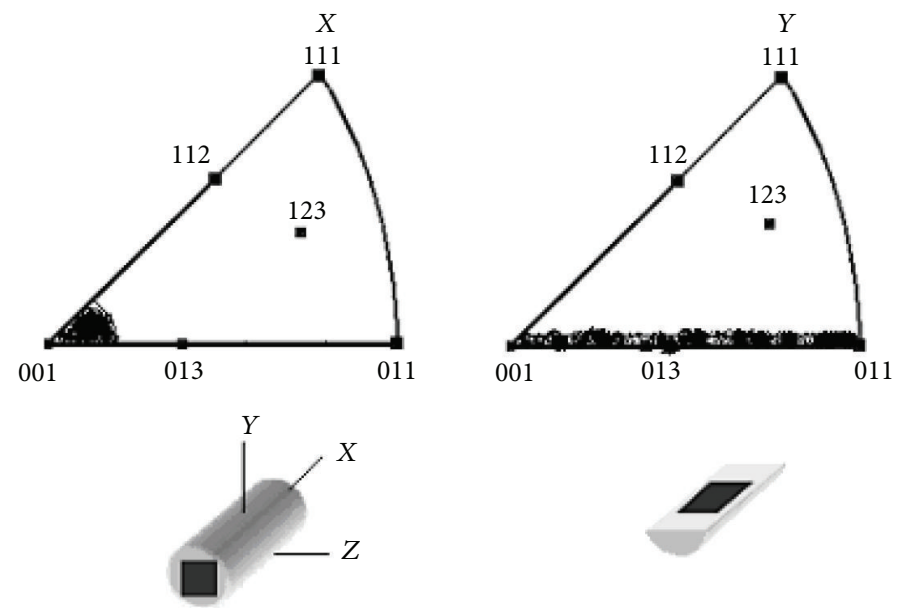

(d)

FIGURE 4: The ideal axial texture with the direction $\langle 100\rangle$ parallel to the extrusion axis. (a) Scheme of orientation distribution crystals when viewed along the direction of extrusion axis; (b) direct pole figure $\{100\}$, on which the axis of sample $(X)$ coincides with extrusion axis; (c) direct pole figure $\{100\}$, on which the sample axis coincides with transverse direction $(Y)$ to the extrusion axis; $(d)$ inverse pole figures of $(X)$ and $(Y)$ directions, respectively; $(Z)$ normal direction to the extrusion axis.

aggregates has axial symmetry, then in order to ease the strain of incompatibility the curling around tension axis can occur (Figure 6) [25].

Formation of axial texture $\{001\}$ was detected in the samples of steel $\mathrm{Fe}-3 \% \mathrm{Si}$ in the process of uniaxial compression at temperatures ranging from $1023 \mathrm{~K}$ to $1173 \mathrm{~K}$ and strain rates of $4.6 \times 10^{-5} \mathrm{~s}^{-1}$ to $5.6 \times 10^{-2} \mathrm{~s}^{-1}$ [26]. It is very likely that the curling is realized at the texture formation during the TE considering experimental pole figures (Figure 1) and microstructure (Figure 2) which is all the more when there is a strong component of torsion at vortex motion of crushed grains, which is to certain extent similar to the turbulent flow of liquid [27]. Such vortex movement of crystallites is displayed in the photos of the microstructure (Figure 3).

3.2. Rolling after TE. Microstructure of the rolled steel after the twist extrusion is shown in Figure 7.

Grain boundaries after rolling with $\varepsilon=0.82$ yet have traces of vortex-strain that are remained from twist extrusion. Rolling along extrusion axis with the reduction of 1.61 resulted in a considerable elongation of the grains in the $\mathrm{RD}$.
Elongation of grains is noticeably smaller when rolling across the TE axis with the reduction of 1.61 .

Figure 8 shows IPFs (ND) after rolling steel samples previously subjected to the TE.

Important components of low-alloyed steel rolling texture are arranged along three fiber orientations [27]:

(1) $\alpha$-fiber: fiber axis $\langle 110\rangle$ parallel to the rolling direction, including the main components of $\{001\}\langle 110\rangle$, $\{112\}\langle 110\rangle$, and $\{111\}\langle 110\rangle$.

(2) $\gamma$-fiber: fiber axis $\langle 111\rangle$ parallel to the normal direction, including the main components of $\{111\}\langle 110\rangle$ and $\{111\}\langle 112\rangle$.

(3) $\varepsilon$-fiber: fiber axis $\langle 110\rangle$ parallel to the transverse direction, including the main components of $\{001\}$ $\langle 110\rangle,\{111\}\langle 112\rangle,\{554\}\langle 225\rangle$, and $\{011\}\langle 100\rangle$.

It can be seen that a typical rolling texture of steel is formed (Figure 8). This texture can be described by two fiber orientations (1) and (2) of the three mentioned above. Let us analyze the volume content of orientations. Let us 


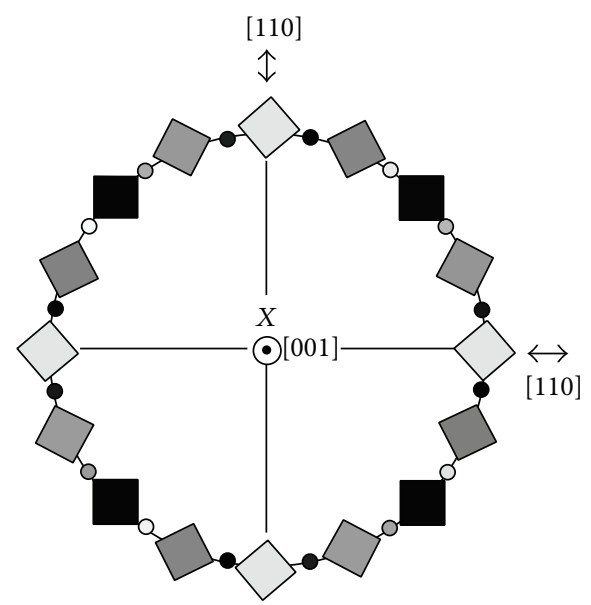

(a)
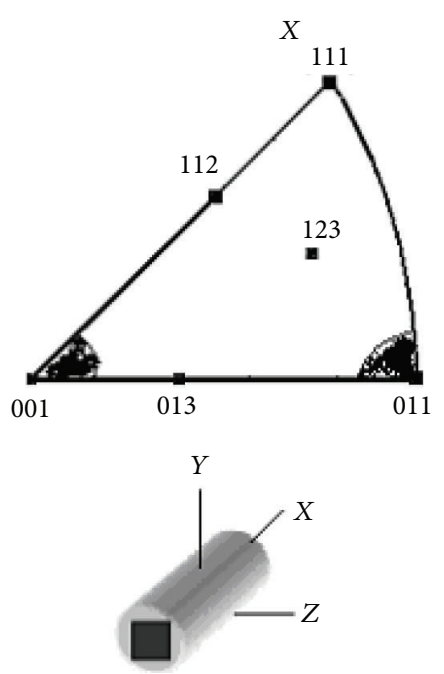

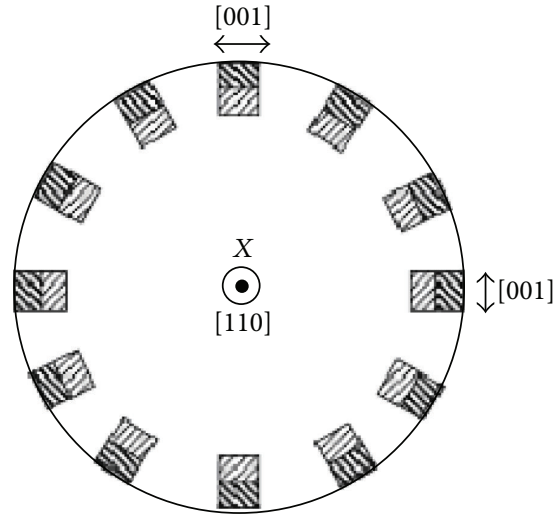

(b)
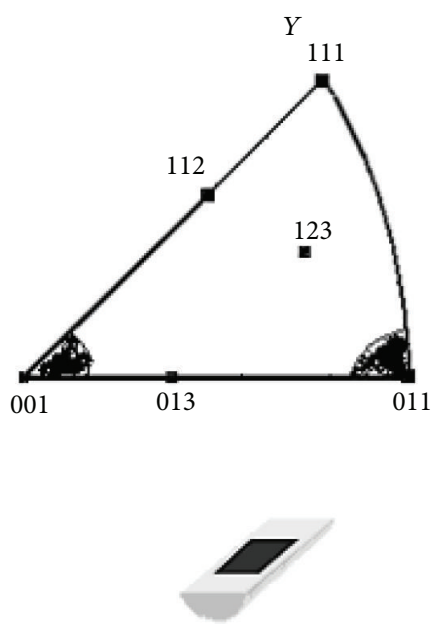

(c)

FIGURE 5: Schematic distribution of crystals in the sample at a cylindrical texture: (a) axis of the sample coincides with the crystallographic axis [001]; (b) axis of the sample coincides with the crystallographic axis [110]; (c) inverse pole figures of $(X)$ and $(Y)$ directions, respectively; $X, Y$, and $Z$ are the same as in Figure 4 .

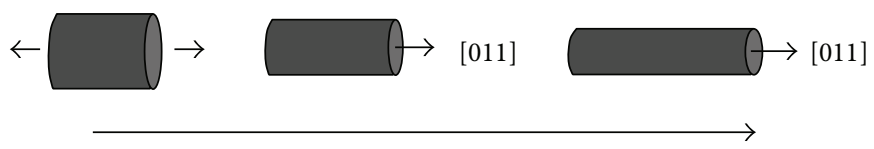

(a)

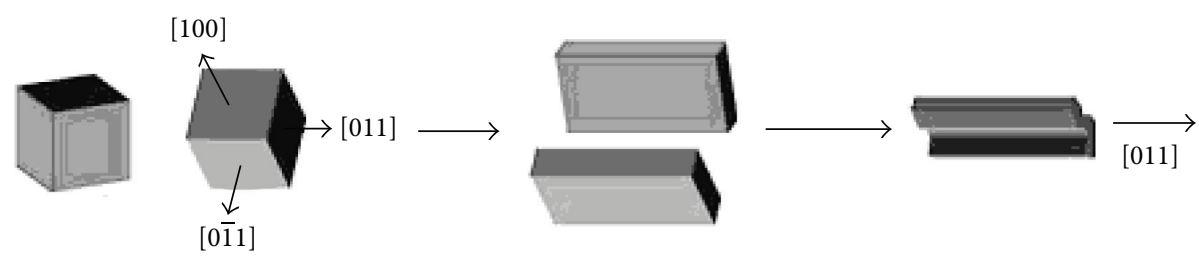

(b)

FIGURE 6: Principle of the curling, which occurs during the axial symmetrical deformation of the sample along $\langle 110\rangle$ axis (a); deformation of the grain (b). 


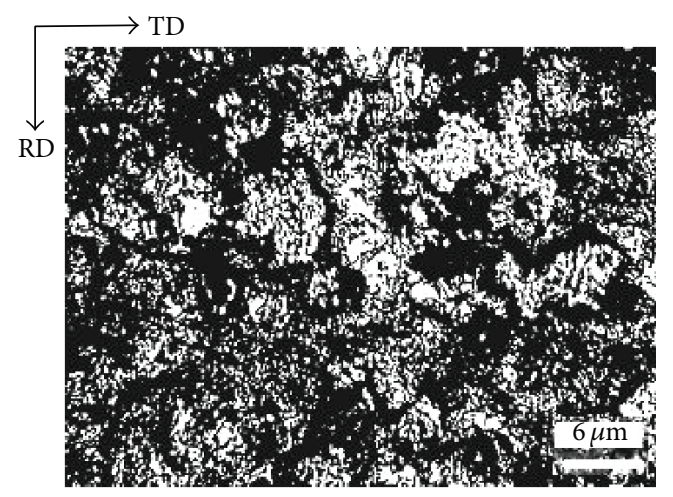

(a)

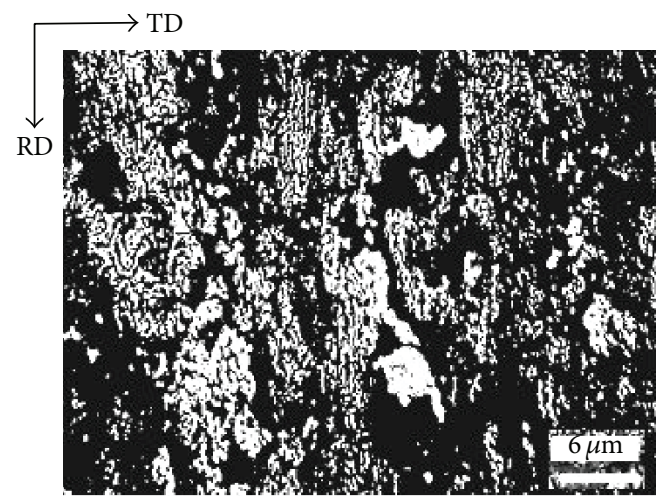

(c)

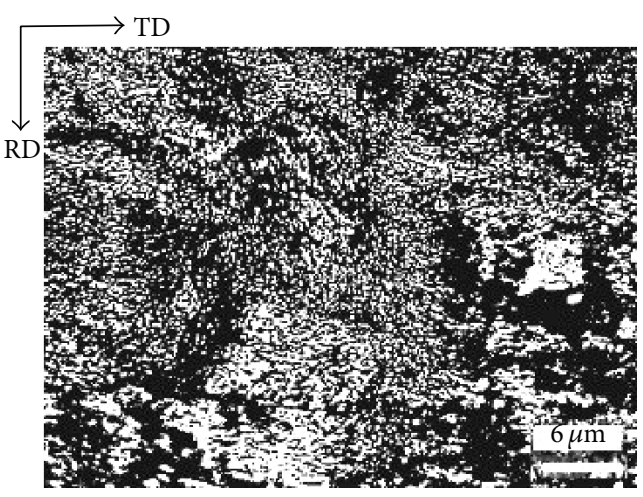

(b)

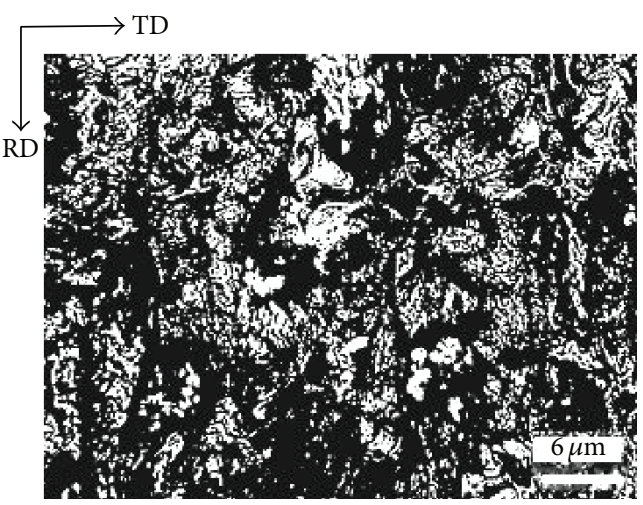

(d)

FiguRE 7: Microstructure of steel rolled after twist extrusion. Rolling along extrusion axis (a, c); rolling transversely to extrusion axis (b, d); $\varepsilon=0.82(\mathrm{a}, \mathrm{b}) ; \varepsilon=1.61(\mathrm{c}, \mathrm{d})$. Photos were made in the rolling plane.

assume that the component composition of the texture of samples is represented by six orientations depicted on IPF ND (Figure 8). Let us assume that the total pole density on the IPF $\mathrm{ND}$ is proportional to the volume content of all orientations and is $100 \%$. Now let us find the volume content of each orientation by ratio

$$
\frac{\Delta V}{V} \cdot 100 \%=\frac{P_{\mathrm{hkl}}}{\sum P_{\mathrm{hkl}}} \cdot 100 \%
$$

Analysis of orientations showed that with increasing of reduction during rolling along $\mathrm{TE}$ axis texture with planes $\{111\}$ parallel to the rolling plane is formed. These components' texture has occupied about $12 \%$ by volume after TE. After rolling with $\varepsilon=0.82$ along TE axis the abovementioned components occupy $14 \%$ of volume, and after rolling with $\varepsilon=$ 1.61 along the TE axis they occupy $30 \%$ of volume (Table 1).

Orientations of crystals with the planes $\{001\}$ parallel to the rolling plane more intensively are formed during the rolling across TE axis. These texture components after TE have occupied about $20 \%$ by volume. After rolling with $\varepsilon=$ 0.82 across TE axis these orientations occupy $32 \%$, and after rolling with $\varepsilon=1.61$ across TE axis they occupy $37 \%$ by volume (Table 2).

Certain differences in mechanisms of plastic deformation cause the difference in the composition of texture components. Texture formation during the rolling along TE
TABLE 1: Volume content of orientations after twist extrusion and subsequent rolling along TE axis.

\begin{tabular}{lccc}
\hline hkl & Twist extrusion & $\begin{array}{c}\text { Consequent } \\
\text { rolling with } \varepsilon \\
=0.82 \text { along } \\
\text { TE axis } \\
\Delta V / V, \%\end{array}$ & $\begin{array}{c}\text { Consequent } \\
\text { rolling with } \varepsilon \\
=1.61 \text { along } \\
\text { TE axis } \\
\Delta V / V, \%\end{array}$ \\
\hline 110 & 24.2 & 12.5 & 8.5 \\
200 & 19.6 & 32.1 & 36.6 \\
211 & 16.4 & 15.3 & 13.9 \\
310 & 15.0 & 15.3 & 6.0 \\
222 & 12.4 & 14.5 & 30.2 \\
321 & 12.3 & 10.3 & 4.9 \\
\hline
\end{tabular}

axis mostly occurs by sliding of dislocations on systems $\{110\}\langle 111\rangle$. During the rolling transversely to the TE axis sliding of dislocations on systems $\{110\}\langle 100\rangle$ prevails [16].

\section{Conclusion}

(1) Double axial cylindrical texture with directions of $\langle 110\rangle$ and $\langle 100\rangle$ parallel to the TE axis is formed during the twist extrusion of low-alloyed steel. 


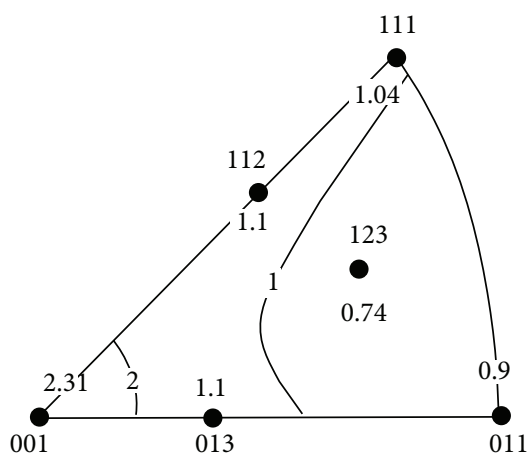

(a)

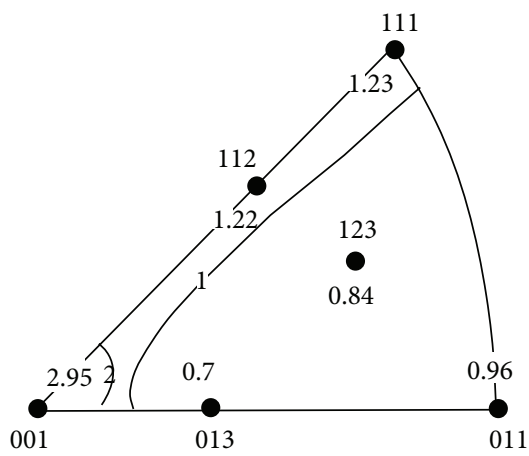

(c)

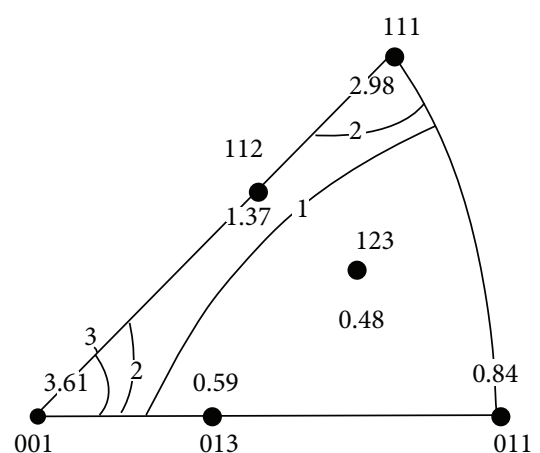

(b)

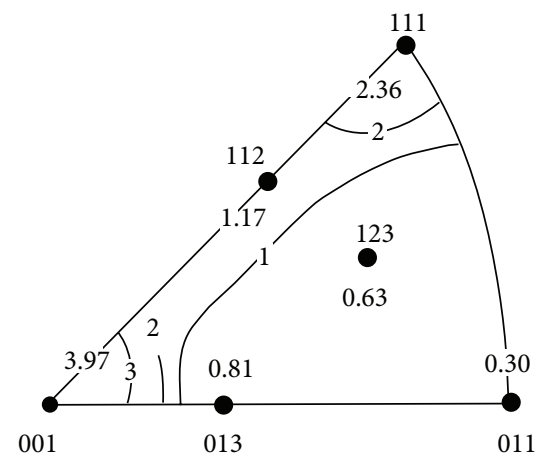

(d)

FIGURE 8: Experimental inverse pole figures of ND of the steel previously subjected to the TE. Rolling along the axis of the TE (a, b). Rolling perpendicular to the TE axis (c, d); $\varepsilon=0.82(\mathrm{a}, \mathrm{c}) ; \varepsilon=1.61(\mathrm{~b}, \mathrm{~d})$.

TABLE 2: Volume content of orientations after twist extrusion and subsequent rolling across TE axis.

\begin{tabular}{lccc}
\hline hkl & Twist extrusion & $\begin{array}{c}\text { Consequent } \\
\text { rolling with } \varepsilon \\
=0.8 \text { across } \\
\text { TE axis } \\
\Delta V / V, \%\end{array}$ & $\begin{array}{c}\text { Consequent } \\
\text { rolling with } \varepsilon \\
=1.61 \text { across } \\
\text { TE axis } \\
\Delta V / V, \%\end{array}$ \\
\hline 110 & 24.1 & 12.2 & 3.2 \\
200 & 20.6 & 37.3 & 43.0 \\
211 & 17.2 & 15.4 & 12.7 \\
310 & 14.4 & 8.9 & 8.8 \\
222 & 11.0 & 15.6 & 25.5 \\
321 & 12.8 & 10.6 & 6.8 \\
\hline
\end{tabular}

(2) Activation of deformation mechanism of the curling induced by the vortex motion of fragments crushed grains promotes the formation of cylindrical axial texture. Such vortex motion of fragments crushed grains in a certain extent is similar to the turbulent fluid flow.

(3) Texture with the components $\{001\}\langle 110\rangle,\{112\}$ $\langle 110\rangle$, and $\{111\}\langle 110\rangle$ is formed during the consequent rolling of the low-alloyed steel after twist extrusion.
(4) Texture components with planes $\{111\}$ that are parallel to the rolling plane are mostly formed with the growth of deformation degree during the subsequent rolling of low-alloyed steel along extrusion axis.

(5) Orientations with planes $\{001\}$ that are parallel to the rolling plane are more intensively formed during the subsequent rolling of steel across extrusion axis.

\section{Competing Interests}

The authors declare that there are no competing interests regarding the publication of this paper.

\section{References}

[1] A. P. Zhilyaev and T. G. Langdon, Bulk Nanostructured Materials: Fundamentals and Applications, John Wiley \& Sons, 2014.

[2] V. V. Stolyarov, Y. E. Beigel'zimer, D. V. Orlov, and R. Z. Valiev, "Refinement of microstructure and mechanical properties of titanium processed by twist extrusion and subsequent rolling," The Physics of Metals and Metallography, vol. 99, no. 2, pp. 204211, 2005.

[3] I. V. Alexandrov, M. V. Zhilina, and J. T. Bonarski, "Formation of texture inhomogeneity in severely plastically deformed copper," Bulletin of the Polish Academy of Sciences: Technical Sciences, vol. 54, no. 2, pp. 199-208, 2006.

[4] I. Alexandrov, J. Bonarski, A. Korshunov, L. Tarkowski, and V. Sitdikov, "Homogeneity of the crystallographic texture and 
deformation behavior in $\mathrm{Cu}$ and $\mathrm{Ti}$ under severe plastic deformation," Archives of Metallurgy and Materials, vol. 53, no. 1, pp. 237-241, 2008.

[5] G. G. Yapici, Severe plastic deformation of difficult-to-work alloys [Master of Science], Texas A\&M University, College Station, Tex, USA, 2004, http://oaktrust.library.tamu.edu/bitstream/handle/ 1969.1/531/etd-tamu-2004A-MEEN-Yapici-1.pdf?sequence=1.

[6] I. J. Beyerlein and L. S. Tóth, "Texture evolution in equalchannel angular extrusion," Progress in Materials Science, vol. 54, no. 4, pp. 427-510, 2009.

[7] M. Salari and J. Kazemi, "Formation of nano-sized grains and textural evolution during accumulative rollbonding (ARB) of Copper sheets," http://www.nanocon.eu/files/proceedings/ nanocon_10/lists/papers/412.pdf.

[8] E. Bonnot, A.-L. Helbert, F. Brisset, and T. Baudin, "Microstructure and texture evolution during the ultra grain refinement of the Armco iron deformed by accumulative roll bonding (ARB)," Materials Science and Engineering A, vol. 561, pp. 60-66, 2013.

[9] V.-D. Cojocaru, D. Raducanu, D. M. Gordin, and I. Cinca, "Texture evolution during ARB (Accumulative Roll Bonding) processing of Ti-10Zr-5Nb-5Ta alloy," Journal of Alloys and Compounds, vol. 546, pp. 260-269, 2013.

[10] B. J. Bonarski, B. Mikułowski, E. Schafler, C. Holzleithner, and M. J. Zehetbauer, "Crystallographic textures of single and polycrystalline pure $\mathrm{Mg}$ and $\mathrm{Cu}$ subjected to HPT deformation," Archives of Metallurgy and Materials, vol. 53, no. 1, pp. 117-123, 2008.

[11] V. V. Usov, M. Salih, Z. Zhong, H.-G. Brokmeier, and N. Schell, "Texture gradient after twist extrusion," in Proceedings of the European XFEL Users' Meeting and Satellite Meetings, DESY, Hamburg, Germany, January 2015, https://www.researchgate .net/publication/273631148_Texture_gradient_after_twist_extrusion.

[12] L.-Z. Wu, J. Chen, Z.-Z. Du, and J.-T. Wang, "Microstructures of ultra-fine grained FeCoV alloys processed by ECAP plus cold rolling and their evolutions during tempering," Transactions of Nonferrous Metals Society of China, vol. 20, no. 4, pp. 602-606, 2010.

[13] S. Ranjbar Bahadori, S. A. A. Akbari Mousavi, and A. R. Shahab, "Sequence effects of twist extrusion and rolling on microstructure and mechanical properties of aluminum alloy 8112," Journal of Physics: Conference Series, vol. 240, no. 1, Article ID 012132, 2010.

[14] K.-T. Park, H.-J. Lee, C. S. Lee, and D. H. Shin, "Effect of postrolling after ECAP on deformation behavior of ECAPed commercial Al-Mg alloy at $723 \mathrm{~K}$, ' Materials Science and Engineering A, vol. 393, no. 1-2, pp. 118-124, 2005.

[15] J. R. Gatti and P. P. Bhattacharjee, "Microstructure and texture of Al-2.5wt.\%Mg processed by combining accumulative roll bonding and conventional rolling," Journal of Materials Engineering and Performance, vol. 23, no. 12, pp. 4453-4462, 2014.

[16] D. V. Gunderov, A. V. Polyakov, I. P. Semenova et al., "Evolution of microstructure, macrotexture and mechanical properties of commercially pure Ti during ECAP-conform processing and drawing," Materials Science and Engineering A, vol. 562, pp. 128136, 2013.

[17] A. Polyakov, D. Gunderov, V. Sitdikov, R. Valiev, I. Semenova, and I. Sabirov, "Physical simulation of hot rolling of ultra-fine grained pure titanium," Metallurgical and Materials Transactions B: Process Metallurgy and Materials Processing Science, vol. 45, no. 6, pp. 2315-2326, 2014.
[18] W. K. Honeycombe, Plastic Deformation of Metals, Edward Arnold, London, UK, 2nd edition, 1984.

[19] N. Shkatulyak, "Effect of twist extrusion and subsequent rolling on the texture and microstructure of aluminium alloy," International Journal of Advances in Materials Science and Engineering, vol. 3, no. 1, pp. 15-25, 2014.

[20] Y. Beygelzimer, D. Orlov, A. Korshunov et al., "Features of twist extrusion: method, structures \& material properties," Solid State Phenomena, vol. 114, pp. 69-78, 2006.

[21] V. Randle and O. Engler, Introduction to Texture Analysis: Macrotexture, Microtexture and Orientation Mapping, CRC Press, London, UK, 2000.

[22] P. R. Morris, "Reducing the effects of nonuniform pole distribution in inverse pole figure studies," Journal of Applied Physics, vol. 30, no. 4, pp. 595-596, 1959.

[23] R. Kaplow, J. F. Peck, F. T. J. Smith, and D. A. Thomas, "Substructure and mechanical properties of refractory metal wires," Technical Documentary Report ML-TDR-64-56, 1964, http://www.dtic.mil/dtic/tr/fulltext/u2/602695.pdf.

[24] S. Leber, "Cylindrical textures in tungsten and other body centered cubic metals," Transactions of the American Society for Metals, vol. 52, pp. 697-713, 1960.

[25] W. Skrotzki, R. Tamm, K. Kegler, and C. G. Oertel, "Deformation and recrystallization textures in iron aluminides," in Proceedings of the International Conference on Microstructure and Texture in Steels and Other Material, pp. 379-390, Jamshedpur, India, February 2008.

[26] Y. Onuki, K. Okayasu, and H. Fukutomi, "Formation of 001 fiber texture in $\mathrm{Fe}-3$ mass\%Si alloy during uniaxial compression deformation at elevated temperatures," ISIJ International, vol. 51, no. 9, pp. 1564-1565, 2011.

[27] Y. Beygelzimer, "Vortices and mixing in metals during severe plastic deformation," Materials Science Forum, vol. 683, pp. 213224, 2011. 

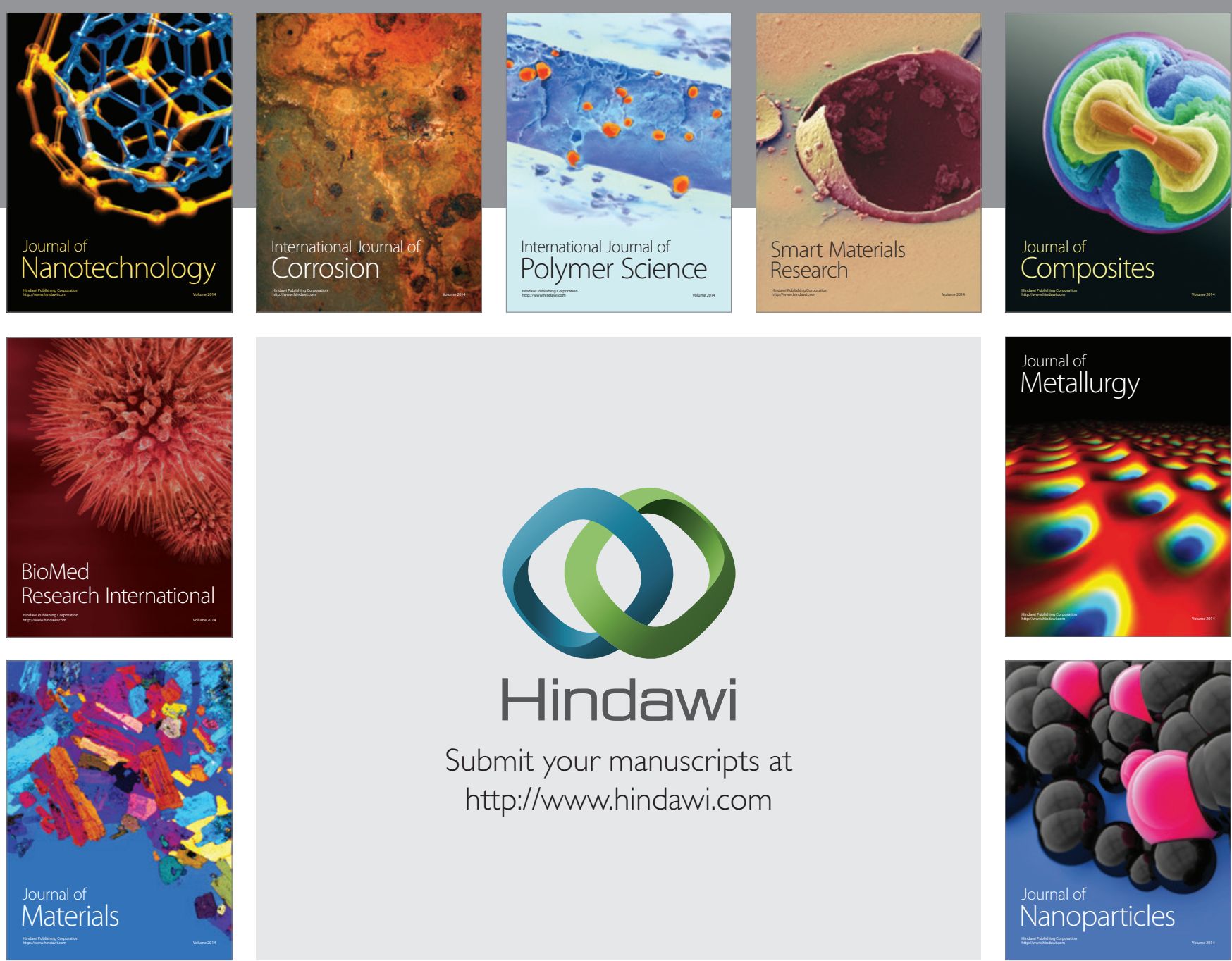

\section{Hindawi}

Submit your manuscripts at

http://www.hindawi.com

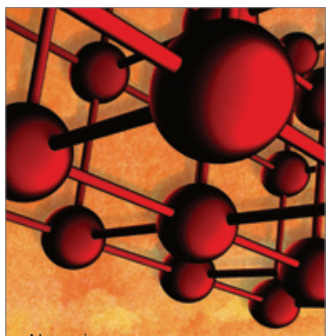

Materials Science and Engineering
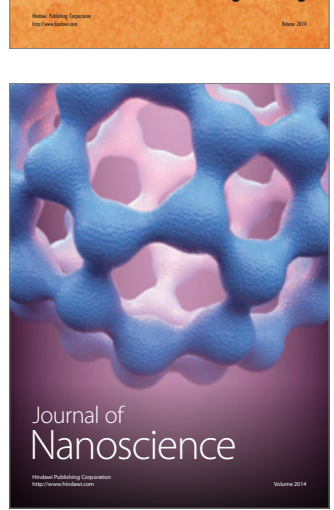
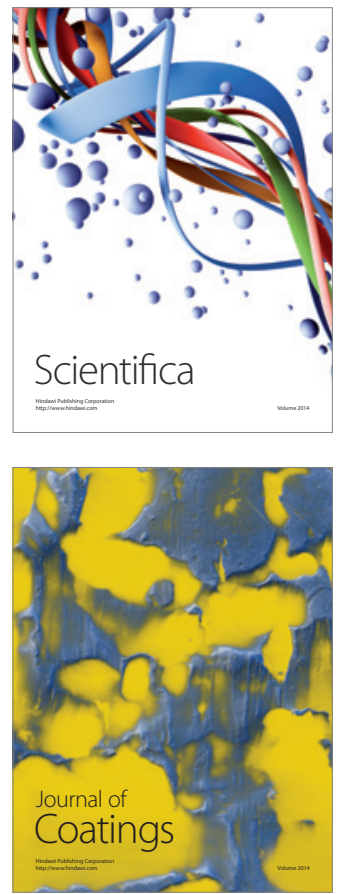
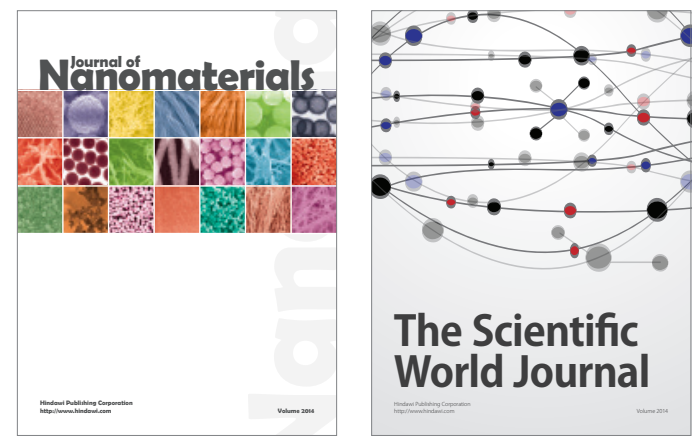

The Scientific World Journal
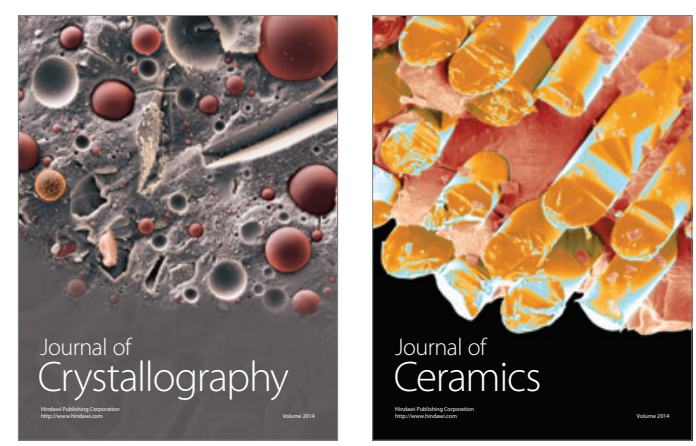
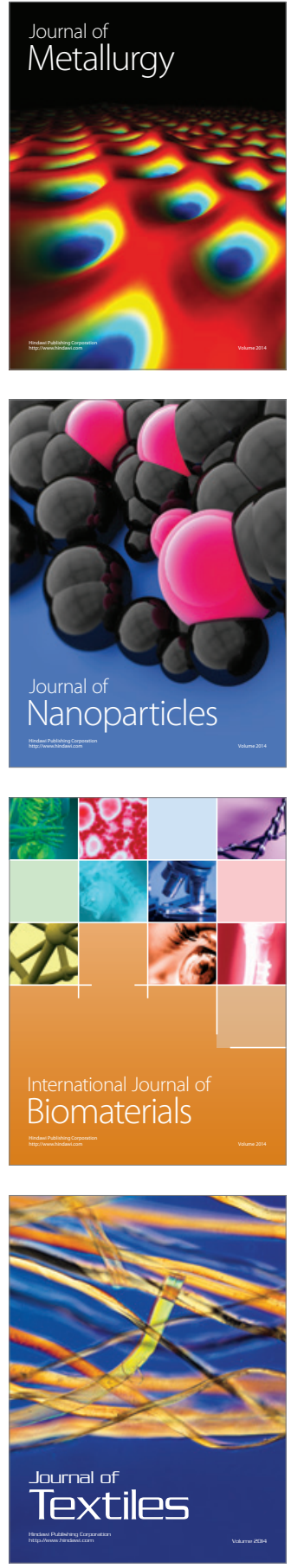